\title{
Multispectral Imaging Fluorescence Microscopy for Living Cells
}

\author{
Yasushi Hiraoka ${ }^{1,2 *}$, Takeshi Shimi ${ }^{1,2}$, and Tokuko Haraguchi ${ }^{1,2}$ \\ ${ }^{1}$ Kansai Advanced Research Center, Communications Research Laboratory, 588-2 Iwaoka, Iwaoka-cho, \\ Nishi-ku, Kobe 651-2492, Japan, and 2 Department of Biology, Graduate School of Science, Osaka University, \\ 1-1 Machikaneyama, Toyonaka, Osaka 560-0043, Japan
}

\begin{abstract}
Multispectral imaging technologies have been widely used in fields of astronomy and remote sensing. Interdisciplinary approaches developed in, for example, the National Aeronautics and Space Administration (NASA, USA), the Jet Propulsion Laboratory (JPL, USA), or the Communications Research Laboratory (CRL, Japan) have extended the application areas of these technologies from planetary systems to cellular systems. Here we overview multispectral imaging systems that have been devised for microscope applications. We introduce these systems with particular interest in live cell imaging. Finally we demonstrate examples of spectral imaging of living cells using commercially available systems with no need for user engineering.
\end{abstract}

Key words: spectroscopy/linear unmixing/GFP/fluorescence resonance energy transfer

Studies of cellular functions are multiparameter problems in nature. In order to understand the cell as an entire system, we need to examine as many parameters as possible within the cell. Genome-wide analysis of expression profiles of genes or proteins, for example, is one of the approaches toward this end. A computational approach of systems biology is another example. Cell biological approaches try to understand dynamic interactions among intracellular molecular components within the spatial and temporal context of the cell, asking how the right molecules come to the right place at the right time. Such a question is not an easy one. Imaging multiple components in living cells can provide a hint for this question. In this context, multispectral imaging has become of increasing importance as an approach to observe dynamics of many proteins within a living cell.

Multispectral imaging is a well-known tool widely used for remote sensing of the Earth's surface from a satellite (NASA, 1973; Curran, 1994; Colarusso et al., 1998). One of

*To whom correspondence should be addressed: Yasushi Hiraoka, Kansai Advanced Research Center, Communications Research Laboratory, 588-2 Iwaoka, Iwaoka-cho, Nishi-ku, Kobe 651-2492, Japan

Tel: +81-78-969-2240, Fax: +81-78-969-2249

E-mail: yasushi@crl.go.jp

Abbreviations: AOTF, acousto-optic tunable filter; CCD, chargecoupled devise; CFP, cyan fluorescent protein; FITC, fluorescein isothiocyanate; FRAP, fluorescence recovery after photobleaching; FRET, fluorescence resonance energy transfer; GFP, green fluorescent protein; LCTF, liquid crystal tunable filter; PMT, photomultiplier tube; UV, ultraviolet; YFP, yellow fluorescent protein. the major methods of multispectral imaging used for remote sensing is Fourier transform spectroscopy (Chamberlain, 1978). In this technology, interferometric measurement of an image generates an interferogram and Fourier transform of the interferogram recovers the spectral distribution of the image. Recently microscope applications of Fourier transform spectroscopy for biological specimens have been made by implementing an interferometer to a fluorescence microscope (Malik et al., 1995; Tsurui et al., 2000). Several other technologies that can be implemented to confocal or widefield fluorescence microscopes have been devised to obtain spectral resolution of microscopic images (Wachman et al., 1997; Ford et al., 2001; Lansford et al., 2001; Haraguchi et al., 2002). Here we introduce these microscopy technologies for multispectral fluorescence imaging with an emphasis on their applications to live cell imaging.

\section{Microscope system designs for multispectral imaging}

An essential part of spectral imaging microscope systems is a spectral dispersion element that separates the incident light into its spectral components. Fig. 1 schematically shows examples of multispectral imaging microscope designs using different types of a spectral dispersion element, such as an interferometer, tunable filter or grating.

Fig. 1A shows a regular widefiled fluorescence microscope with a set of three optical elements, that is, an excita- 
A

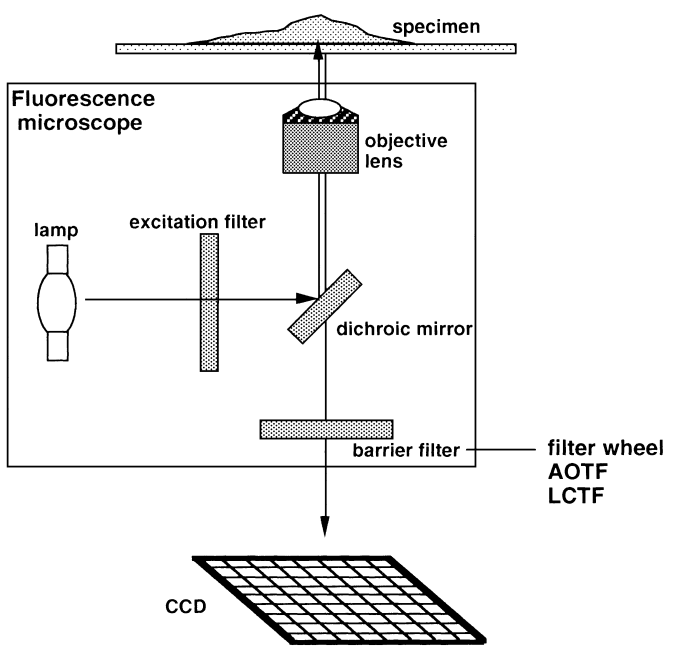

B

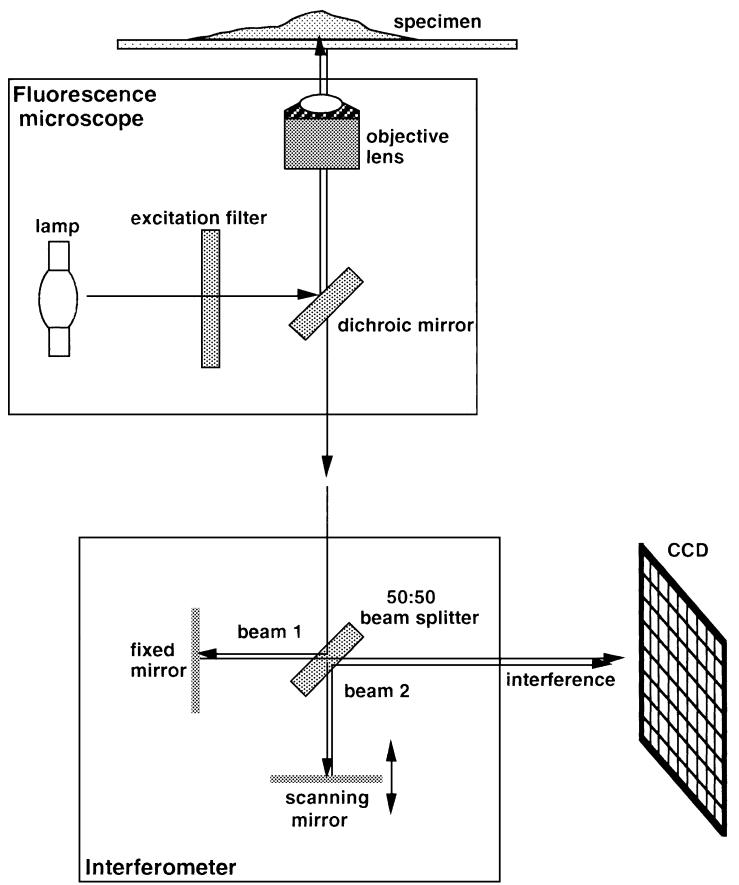

C

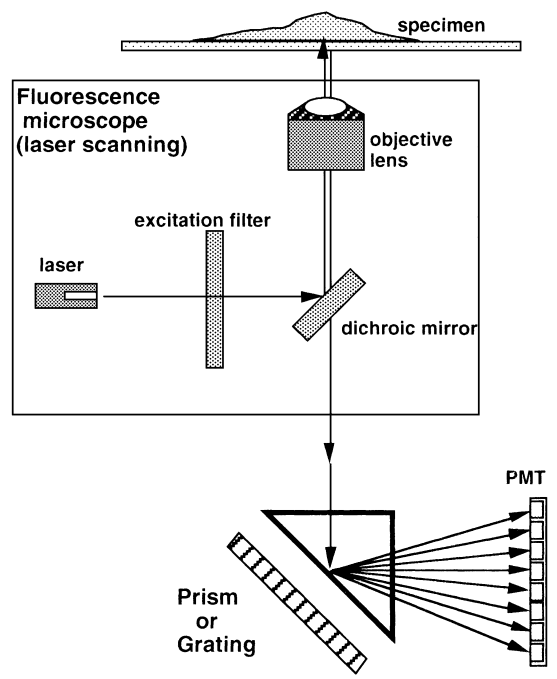

D

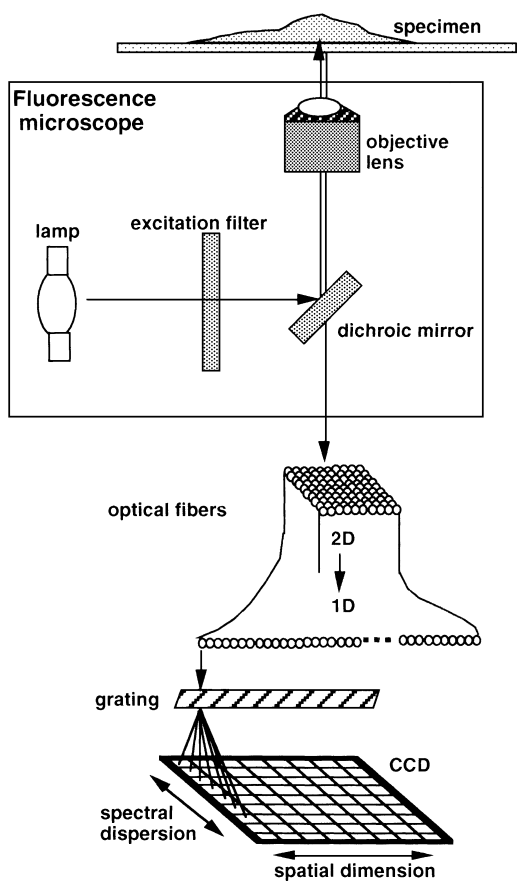

Fig. 1. Microscope systems for multispectral imaging. (A) Filter-based spectral imaging. (B) Fourier transform-based interferometric image spectroscopy. The diagram merely shows the principle of an interferometer, but not exact light paths. (C) Grating-based spectral imaging using a laser scanning confocal microscope. (D) Grating-based spectral imaging using a widefield fluorescence microscope with an optical fiber coupling.

tion filter, a barrier filter and a dichroic beam splitter. A standard filter combination is designed for a single range of wavelength to pass these optical elements. On the other hand, in order to obtain multispectral images with no filter changes, multi-bandpass filters which pass multiple wave- length regions are used for an excitation filter, a barrier filter and a dichroic beam splitter. For switching wavelengths, a set of discrete filters are mounted on a revolving wheel and filters are switched by revolving the wheels which are placed at the position of the excitation filter and the barrier 
filter (Hiraoka et al., 1991; Haraguchi et al., 1999). Although wavelength switching is relatively slow, a discrete set of multispectral images can be obtained in principle by switching filters by revolving filter wheels. Alternatively, for quick switching of wavelength, a single tunable filter such as acousto-optic tunable filter (AOTF) or liquid crystal tunable filter (LCTF) is inserted at the barrier filter position. AOTF and LCTF crystals can provide continuous tunability of light wavelength to transmit with a narrow bandwidth (Morris et al., 1994; Wachman et al., 1997; Lansford et al., 2001). Wavelength switching of LCTF is slower than that of AOTF, but rejection of out-of-band transmission is higher in LCTF than in AOTF (out-of-band transmission relative to the peak transmission: less than $10^{-4}$ in LCTF compared with $10^{-2}$ to $10^{-3}$ in AOTF). Such a tunable filter is placed at the position of the regular optical filters in confocal or widefiled fluorescence microscopes. An application of AOTF in a widefiled fluorescence microscope is described in Wachman et al. (1997); an application of LCTF to a laser scanning confocal microscope with two-photon excitation is described in Lansford et al. (2001). These filters can be inserted to a widefield fluorescence microscope, a singlephoton or two-photon confocal microscope.

An example in Fig. 1B shows a microscope system using an interferometer for spectral dispersion. Fourier transform spectroscopy is based on an interferometer (Malik et al., 1995; Schröck et al., 1996). An interferometer is attached to a regular widefield fluorescence microscope. The interferometer divides the fluorescence light coming out from the microscope into two beams. Optical path difference is generated between the two beams by varying the optical path length of one of the beams by moving a scanning mirror. The beams are then recombined to interfere with each other. Interference intensity as a function of optical path difference (interferogram) is recorded on a two-dimensional, solidstate detector (typically CCD). Spectral information of fluorescence images is recovered by taking Fourier transform of the interferogram. Mathematical details of Fourier transform-based interferometric spectroscopy are described previously (Chamberlain, 1978; Garini et al., 1996). Multispectral imaging microscope systems based on interferometric spectroscopy was successfully applied to karyotyping of human chromosomes (Schröck et al., 1996; Garini et al., 1996). In these studies, multispectral images of chromosomes that are stained with combinations of fluorescent dyes are spectrally resolved to distinguish 24 different types of the chromosome simultaneously.

An example shown in Fig. 1C uses a grating or prism as a spectral dispersion element. Point measurement of fluorescence separated by a grating or prism generates a linear array of spectral components. This method can be ready implemented to a laser scanning confocal microscope. Spectrally-resolved fluorescence is detected by linearly scanning a single photomultiplier tube (PMT) as a detector,
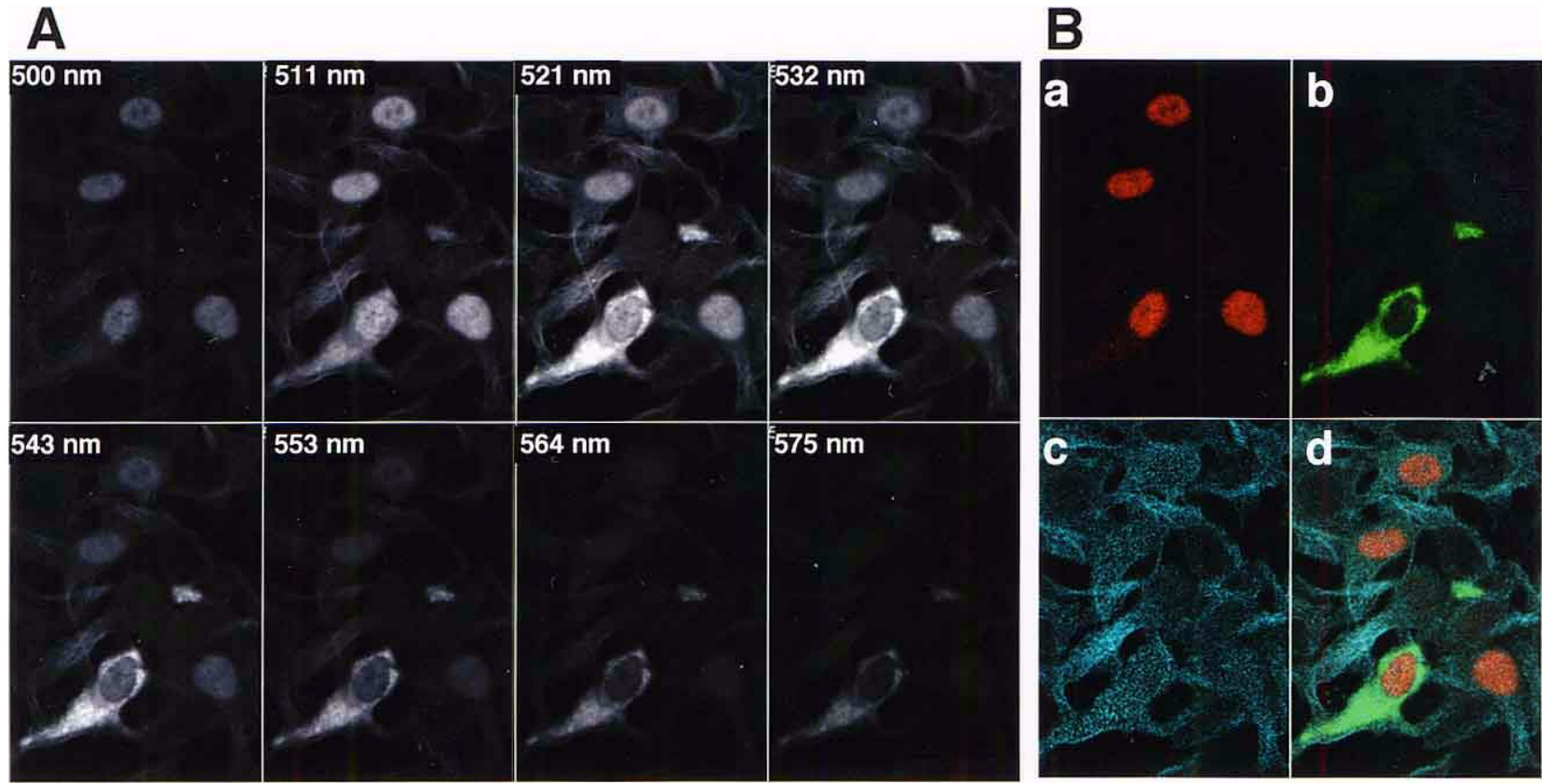

Fig. 2. Multispectral images and linear unmixing. (A) Multispectral images of HeLa cells. HeLa cells expressing histone H2B-GFP, YFP-RanGAP1 were fixed with $3.7 \%$ formaldehyde, then stained with an anti- $\alpha$-tubulin antibody and detected with an FITC-conjugated secondary antibody. The cells were excited by $488 \mathrm{~nm}$ light from an argon laser, and observed using a water-immersion objective lens (C-Apochromat $40 \times / \mathrm{NA}=1.2)$ and a dichroic mirror (HFT413/488). (B) Images of GFP (a), YFP (b) and FITC (c) was separated by linear unmixing, and merged with pseudocolor (d). Images were obtained using a commercially available multispectral microscope (Zeiss LSM510 META), and processed with its standard software package of linear unmixing. 
or detected directly on a linear array of PMTs, placed at the confocal image plane. Point measurement is repeated pixel by pixel during laser scanning to generate a set of twodimensional images along the wavelength. Such microscope systems for spectral imaging are now commercially available. Zeiss LSM510 META uses a grating as a spectral dispersion element and an array of PMT as a detector (Haraguchi et al., 2002). Leica TCS SP2 AOBS uses a prism as a spectral dispersion element and a scanning PMT as a detector.

The last example shown in Fig. 1D is based on a grating in combination with a widefield fluorescence microscope. This microscope design has a unique feature in which a microscopic image is captured on a two-dimensional array of optical fibers as an image detector, which is converted to a one-dimensional array at the opposite end of the optical fiber bundle; the fluorescent light coming out from each of the fibers is spectrally dispersed by grating optics to generate a two-dimensional image, that is, one spatial dimension and one spectral dimension (Matsuoka et al., 2002). This device is potentially interesting, but a current technological limitation is the spatial resolution limited by the distance between neighboring optical fibers on the light-receiving surface. The distance between fibers used in the report (Matsuoka et al., 2002) is $230 \mu \mathrm{m}$. Thus, the resolution is considerably lower than that of the CCD (the pixel size of a typical CCD chip for a microscopic use is about $10 \mu \mathrm{m}$ ). Fabrication of a finer optical fiber coupling is awaited to improve the resolution of this device.

\section{Linear unmixing of multispectral images}

Multispectral imaging fluorescence microscopy of biological specimens provides the versatility in combination of fluorescent dyes. Multispectral images are obtained as a set of two-dimensional images as a function of wavelength (Fig. 2A). This example shows fixed HeLa cells that are stained with GFP, FITC, and YFP, which have fluorescence

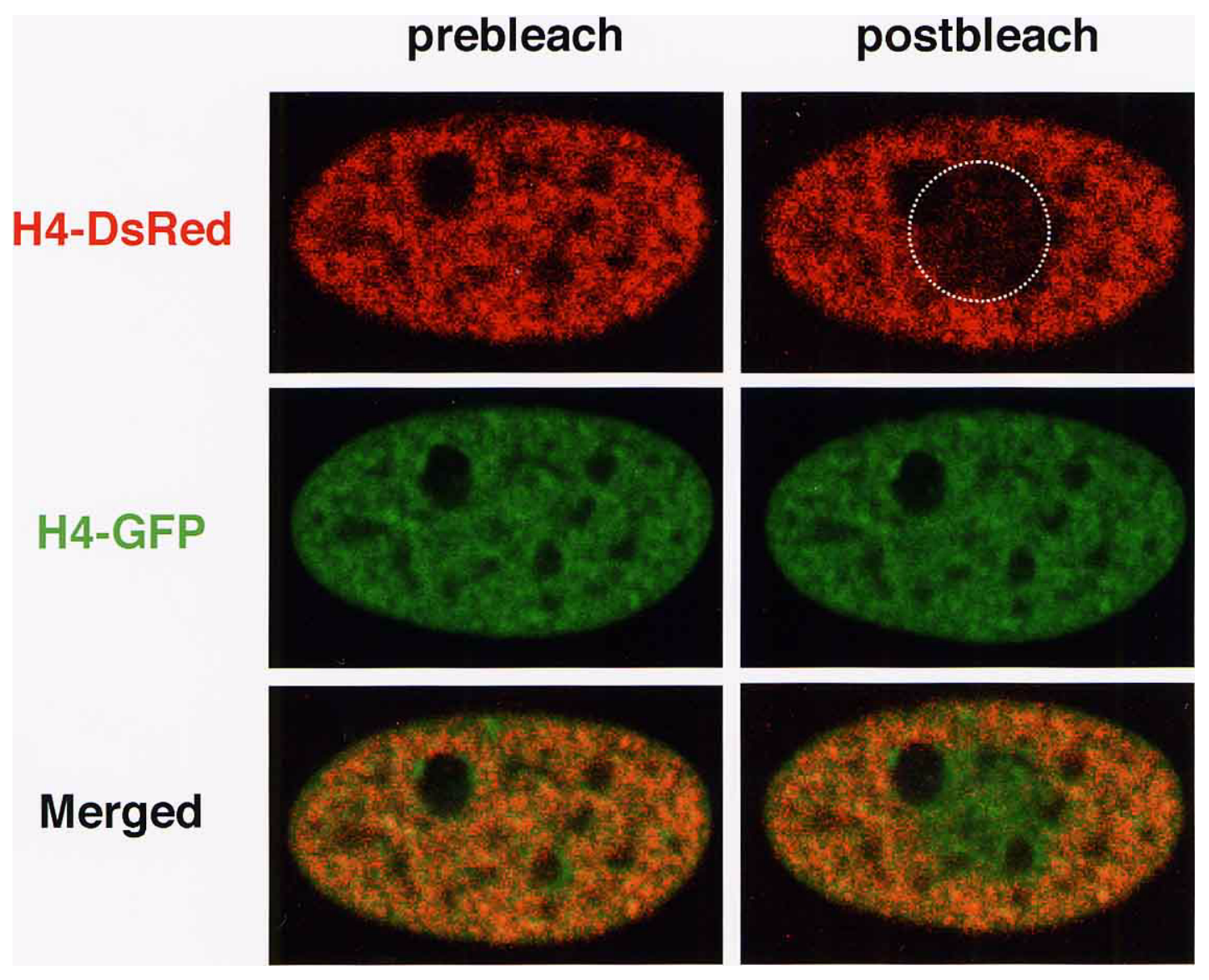

Fig. 3. FRAP analysis in multiply-stained cells. In HeLa cells expressing DsRed fusion and GFP-fusion of histone H4 (H4-DsRed and H4-GFP, respectively), H4-DsRed displayed in red was photobleached by the $543 \mathrm{~nm}$ light from a helium-neon laser (upper right panel; area indicated by the circle) without photobleaching fluorescence of H4-GFP displayed in green (middle row) using an oil-immersion objective lens (Plan-Apochromat 63×/NA=1.4) and a dichroic mirror (HFT488/543). In the merged image, bleached area is seen in green within the non-bleached area seen in yellow. The second non-bleached structure acts as a structural marker in following behaviors of proteins in the bleached area. 
spectra closely overlapping with each other (the emission peak is $509 \mathrm{~nm}, 518 \mathrm{~nm}$ and $527 \mathrm{~nm}$, respectively). Spectral overlap between fluorescent dyes can be separated into spectra of each dye by computational processing called "linear unmixing" (Tsurui et al., 2000; Lansford et al., 2001; Haraguchi et al., 2002).

Fluorescence images of a multiply-stained specimen are obtained as a linear combination of fluorescent dyes used to stain the specimen (linear mixing). By using the spectrum of each dye, observed spectra can be separated to the contribution of each dye (linear unmixing). This is described as:

$$
\mathrm{I}(\lambda)=\Sigma_{\mathrm{i}} \mathrm{A}_{\mathrm{i}} \mathrm{R}_{\mathrm{i}}(\lambda)
$$

where $I(\lambda)$ is the measured spectrum as a function of wavelength $\lambda$, coefficients $\mathrm{A}_{\mathrm{i}}$ are the contribution of the $i$-th dye, and $\mathrm{R}_{\mathrm{i}}(\lambda)$ is reference spectrum of the $i$-th dye. The coefficients $A_{i}$ are determined with least square fitting:

$$
\Sigma_{\mathrm{j}}\left\{\mathrm{I}\left(\lambda_{\mathrm{j}}\right)-\Sigma_{\mathrm{i}} \mathrm{A}_{\mathrm{i}} \mathrm{R}_{\mathrm{i}}\left(\lambda_{\mathrm{j}}\right)\right\}^{2}<\min
$$

that minimizes the square difference between measured and calculated spectra, that is,

$$
\frac{\partial}{\partial \mathrm{A}_{\mathrm{i}}} \Sigma_{\mathrm{j}}\left\{\mathrm{I}\left(\lambda_{\mathrm{j}}\right)-\Sigma_{\mathrm{i}} \mathrm{A}_{\mathrm{i}} \mathrm{R}_{\mathrm{i}}\left(\lambda_{\mathrm{j}}\right)\right\}^{2}=0
$$

therefore,

$$
\Sigma_{\mathrm{j}} \mathrm{R}_{\mathrm{i}}\left(\lambda_{\mathrm{j}}\right)\left\{\mathrm{I}\left(\lambda_{\mathrm{j}}\right)-\Sigma_{\mathrm{i}} \mathrm{A}_{\mathrm{i}} \mathrm{R}_{\mathrm{i}}\left(\lambda_{\mathrm{j}}\right)\right\}=0
$$

The equations are usually solved by singular value decomposition (Tsurui et al., 2000; Lansford et al., 2001).

This method is capable of separating GFP, YFP and FITC as shown in Fig. 2. Best results of color separation were obtained when cells stained with each one of the dyes were used to measure a reference spectrum. In our experiments, the use of spectra measured in solution resulted in residual crossover of spectra. This capability significantly increases the versatility of choice of fluorescence dyes that can be used for staining cells.

\section{Biological applications for live cell imaging}

Temporal resolution of multispectral images is crucial in observation of living cells. Multispectral imaging for a limited number of wavelengths can be accomplished by rapidly switching discrete filters. Temporal resolution in filter-based spectral imaging microscope systems is limited by the speed of wavelength switching, which is on an order of seconds with a filter wheel, an order of a few 10 milliseconds with LCTF, and submilliseconds with AOTF. By the use of a grating or prism, information of continuous spectra

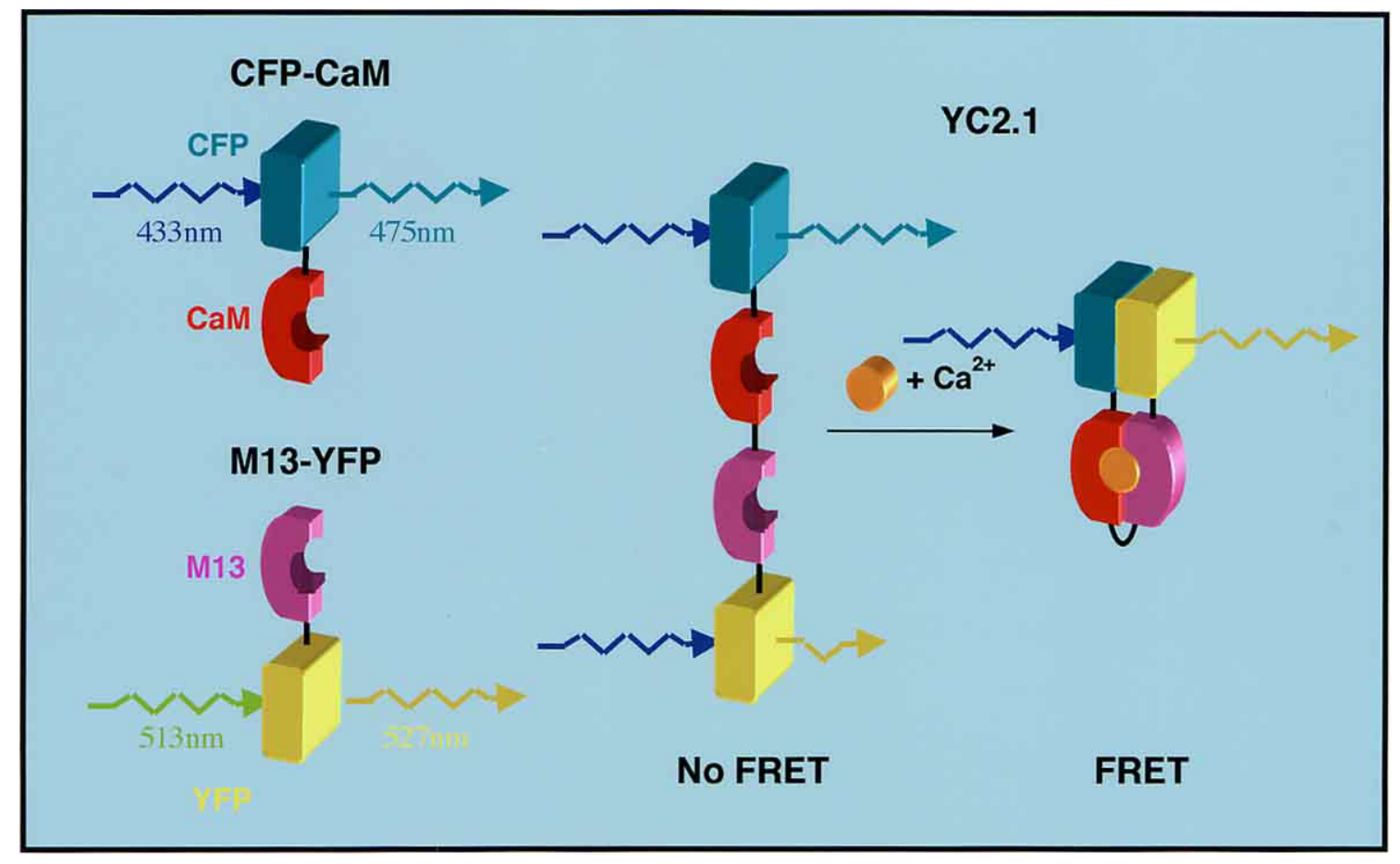

Fig. 4. Conformational change of YC2.1 in the presence of $\mathrm{Ca}^{2+}$ generates FRET signal. The joint version of YC2.1 is composed of CFP and YFP connected by calmodulin (CaM) and M13 (Miyawaki et al., 1997; Miyawaki et al., 1999). Binding of Ca ${ }^{2+}$ to YC2.1 causes conformational change by interaction between the CaM portion and the M13 portion, bringing CFP and YFP in close proximity to generate FRET. 
can be obtained simultaneously with no moving elements. Grating-based spectral imaging microscope systems provide simultaneous spectral dispersion, and thus is useful for multispectral imaging of rapid processes in living cells.
Simultaneous multispectral imaging is useful in applications of fluorescence recovery after photobleaching (FRAP) to measure the mobility of proteins in living cells. In FRAP experiments, fluorescently-tagged protein expressed in liv-

A

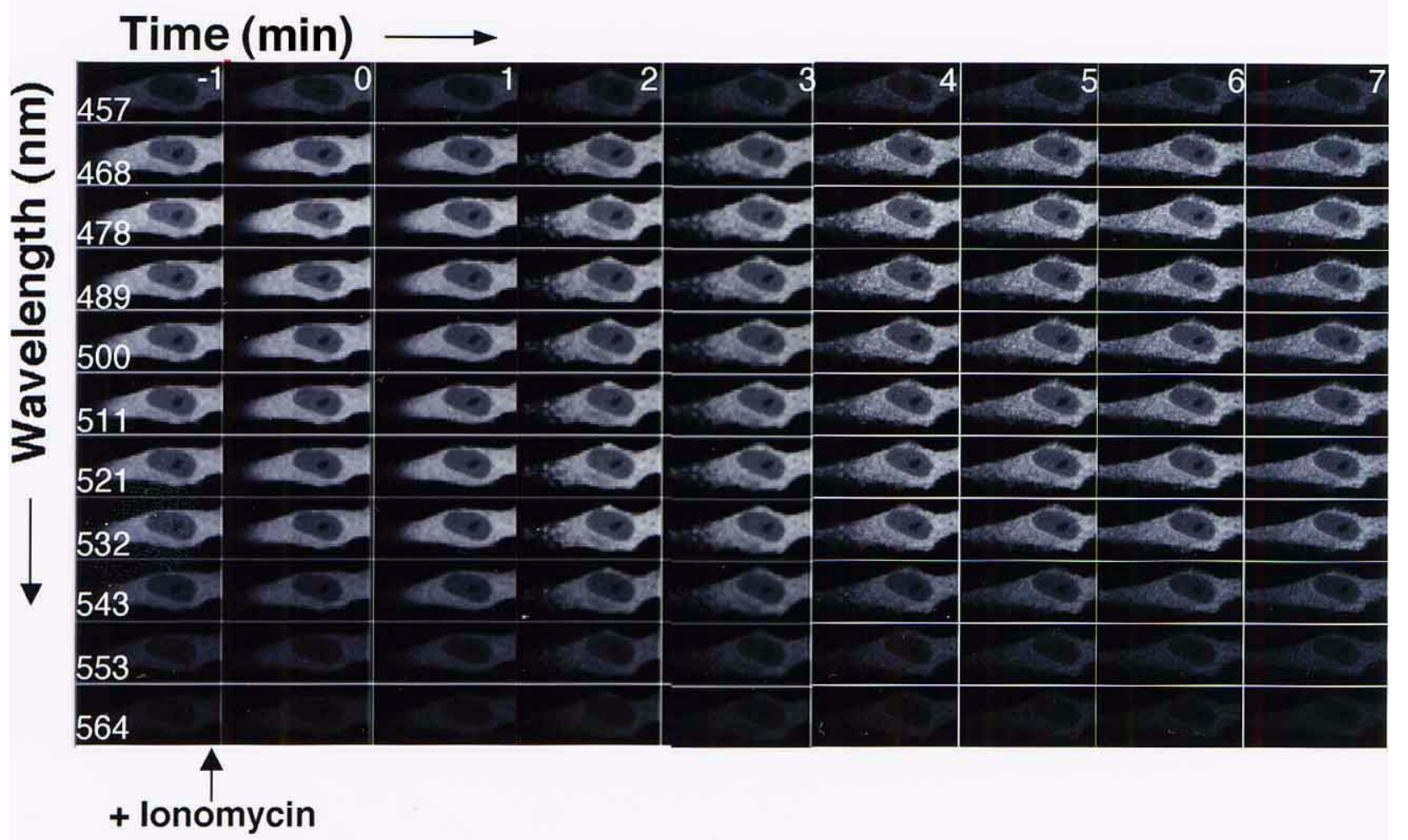

B

time

\section{lonomycin $-(0 \mathrm{sec})$}

$+(100 \mathrm{sec})$
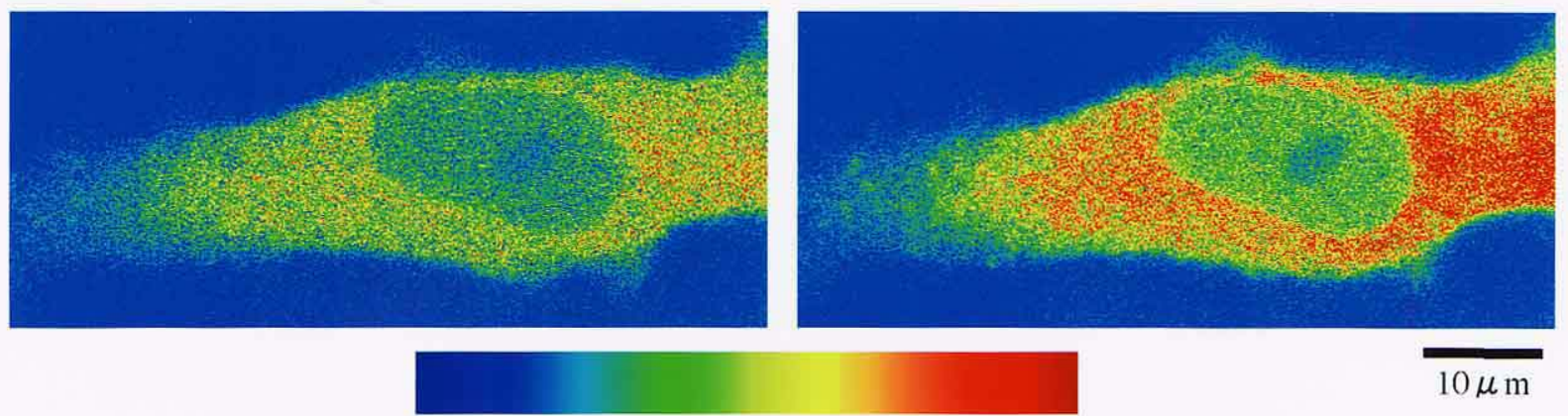

$10 \mu \mathrm{m}$

\section{low}

high

Fig. 5. FRET images of YC2.1. (A) Temporal series (from left to right) of spectral images (from top to bottom) of living HeLa cells expressing YC2.1. The cells were excited by the light of $413 \mathrm{~nm}$ wavelength from a UV argon laser (Coherent, Santa Clara, CA), and observed using a water-immersion objective lens (C-Apochromat 63×/NA=1.2) and a dichroic mirror (HFT413). (B) Ratio image of fluorescence intensity at $532 \mathrm{~nm}$ to that at $478 \mathrm{~nm}$ is displayed for before and after the addition of ionomycin. 
ing cells is photobleached in a limited area, and the rate of recovery is followed to estimate the mobility of the protein. It is often convenient if another structure is also stained in the same cell as the FRAP area can be compared relative to this structure as a reference (Fig. 3).

Multispectral imaging is also powerful to detect spectral changes associated with fluorescence resonance energy transfer (FRET). For FRET measurement we used yellow cameleon-2 (YC2.1) as a FRET-based calcium indicator dye (Miyawaki et al., 1997; Miyawaki et al., 1999). YC2.1 is composed of CFP and YFP as a donor and an acceptor of FRET, respectively (Fig. 4). This molecule undergoes a conformational change in the presence of $\mathrm{Ca}^{2+}$, bringing CFP and YFP in close proximity to generate FRET (see legend to

A

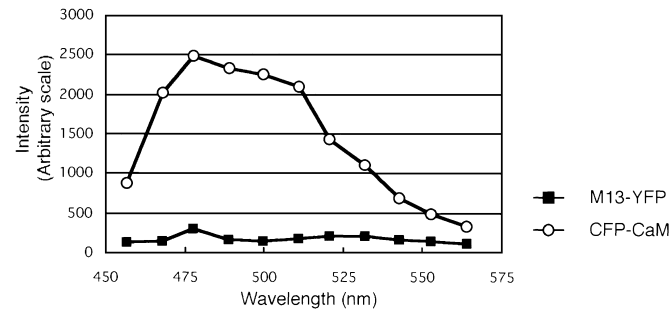

B

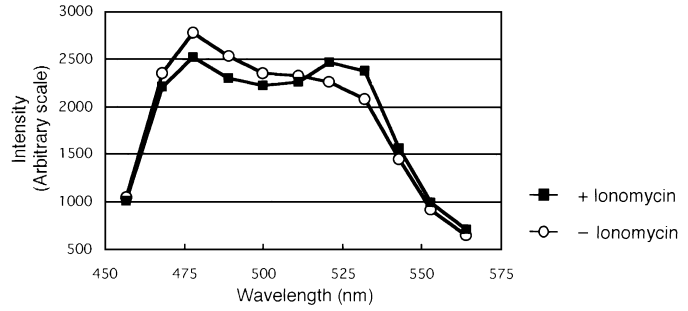

C

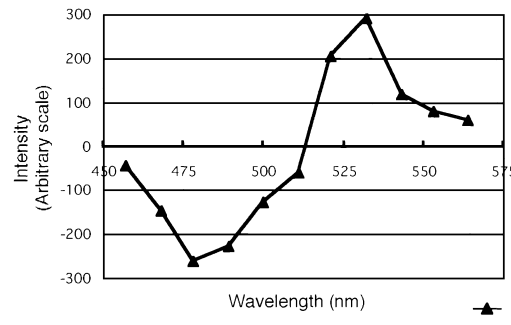

D

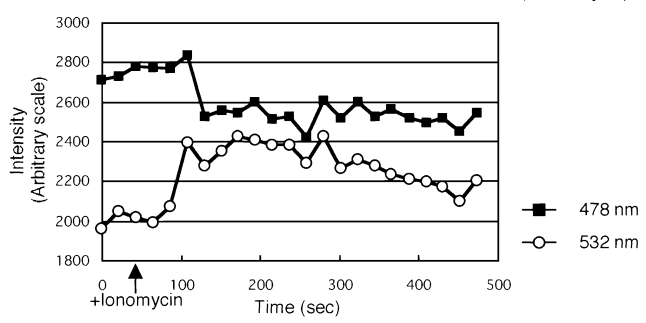

Fig. 6. Spectral change of YC2.1. (A) Fluorescence spectra of CFP-CaM (filled square) and M13-YFP (open circle) measured in HeLa cells excited by $413 \mathrm{~nm}$ light. (B) Fluorescence spectra of YC2.1, excited by $413 \mathrm{~nm}$ light, before (open circle) and after (filled square) ionomycin treatment of HeLa cells. (C) FRET spectra, subtracting fluorescence spectra before ionomycin treatment from that after ionomycin treatment. (D) Fluorescence intensity at $478 \mathrm{~nm}$ and $532 \mathrm{~nm}$ is plotted as a function of time. The arrow indicates the time of addition of ionomycin.
Fig. 4). To detect FRET in living HeLa cells, YC2.1 was introduced into the cells, and calcium uptake to the cells was induced by the addition of ionomycin. Previously we used $458 \mathrm{~nm}$ excitation from an argon laser, one of the standard laser sources of Zeiss LSM510 META (Haraguchi et al., 2002). Obviously this wavelength is not optimal to excite CFP. Thus, in this report, we used $413 \mathrm{~nm}$ excitation light from a UV argon laser added to this microscope system. A blue diode laser emitting $405 \mathrm{~nm}$ is another possible choice for CFP/YFP FRET.

Figure 5 shows a FRET-associated spectral change of YC2.1 induced by changes in the intracellular calcium concentration in HeLa cells. The cells were excited by the excitation light of $413 \mathrm{~nm}$ wavelength. Images were obtained as a temporal series of spectral images (Fig. 5A); fluorescence intensity is displayed in Fig. 5B as a pseudocolor representation. As a control, HeLa cells expressing either CFP-CaM or M13-YFP were excited at the wavelength of $413 \mathrm{~nm}$. Basically no fluorescence was detected from M13-YFP excited at $413 \mathrm{~nm}$ (Fig. 6A). Fluorescence of YC2.1 before the addition of ionomycin was basically that of CFP-CaM (compare Fig. 6B with Fig. 6A), and changed its spectra shortly after the addition of ionomycin (Fig. 6B and C). In Fig. 6D, fluorescence intensities at $478 \mathrm{~nm}$ and at $532 \mathrm{~nm}$ are plotted as a function of time; the acceptor signal (at 532 $\mathrm{nm}$ ) increased and the donor signal (at $478 \mathrm{~nm}$ ) decreased upon the induction, indicating that FRET occurred. These results demonstrate an advantage of spectral imaging in which spectral changes can be directly measured at multiple wavelengths.

\section{Concluding remarks}

The temporal and spectral resolution of fluorescence microscope images provides a unique opportunity to detect spectral changes of fluorescence in living cells. Capability of spectral imaging will provide the impetus for the development of a new class of fluorescent dyes. Dyes that change their spectra under certain conditions (e.g. phosphorylation and dephosphorylation events, molecular interactions or conformational change of proteins) are also awaited for applications in spectral imaging to detect changes in the intracellular environment. The microscopy technology of multispectral imaging could prove useful for the real-time detection of biochemical reactions inside single cells. Technologies for microscope image acquisition, processing, and analysis are based on many disciplines. Interdisciplinary approaches in chemistry, material science and engineering will lead to further technological development of live cell imaging.

Acknowledgments. We would like to thank Dr. Atsushi Miyawaki for the yellow cameleon dyes, and Dr. Hiroshi Kimura for the histone H4 fusion constructs. This work was supported by a grant from the Japan Science and Technology Corporation. 


\section{References}

Boardman, J.W. 1989. Inversion of imaging spectrometry data using singular value decomposition. Proc. IGARSS'89 Sensing, 4: 2069-2072.

Chamberlain, J. 1978. The Principles of Interferometric Spectroscopy. Wiley, New York.

Colarusso, P., Kidder, L.H., Levin, I.W., Fraser, J.C., Arens, J.F., and Lewis, E.N. 1998. Infrared spectroscopic imaging: From planetary to cellular systems. Appl. Spectrosc., 52: 106A-120A.

Curran, P.J. 1994. Imaging spectrometry. Prog. Phys. Geog., 18: 247-266.

Ford, B.K., Volin, C.E., Murphy, S.M., Lynch, R.M., and Descour, M.R. 2001. Computed tomography-based spectral imaging for fluorescence microscopy. Biophys. J., 80: 986-993.

Garini,Y., Macville, M., du Manoir, S., Buckwald, R.A., Lavi, M., Katzir, N., Wine, D., Bar-Am, I., Schröck, E. Cabib, D., and Ried, T. 1996. Spectral karyotyping. Bioimaging, 4: 65-72.

Haraguchi, T., Ding, D.-Q., Yamamoto, A., Kaneda, T., Koujin, T., and Hiraoka, Y. 1999. Multiple-color fluorescence imaging of chromosomes and microtubules in living cells. Cell Struct. Funct., 24: 291-298.

Haraguchi, T., Shimi, T., Koujin, T., Hashiguchi, N., and Hiraoka, Y. 2002. Spectral imaging for fluorescence microscopy. Genes Cells, 7: 881-887.

Hiraoka, Y., Swedlow, J.R., Paddy, M.R., Agard, D.A., and Sedat, J.W. 1991. Three-dimensional multiple-wavelength fluorescence microscopy for the structural analysis of biological phenomena. Semin. Cell Biol., 2: 153-165.

Lansford, R., Bearman, G., and Fraser, S.E. 2001. Resolution of multiple green fluorescent protein color variants and dyes using two-photon microscopy and imaging spectroscopy. J. Biomed. Opt., 6: 311-318.

Malik, Z., Cabib, D., Buckwald, R.A., Talmi, A., Garini, Y., and Lipson,
S. G. 1996. Fourier transform multipixel spectroscopy for quantitative cytology. J. Microsc., 182: 133-140.

Matsuoka, H., Kosai, Y., Saito, M., Takeyama, N., and Suto, H. 2002. Single-cell viability assessment with a novel spectro-imaging system. J. Biotech., 94: 299-308.

Miyawaki, A., Llopis, J., Heim, R., McCaffery, J.M., Adams, J.A., Ikura, M., and Tsien, R.Y. 1997. Fluorescent indicators for $\mathrm{Ca}^{2+}$ based on green fluorescent proteins and calmodulin. Nature, 388: 882-887.

Miyawaki, A., Griesbeck, O., Heim, R., and Tsien, R.Y. 1999. Dynamic and quantitative $\mathrm{Ca}^{2+}$ measurements using improved cameleons. Proc. Natl. Acad. Sci. USA, 96: 2135-2140.

Morris, H.R., Hoyt, C.C., and Treado, P.J. 1994. Imaging spectrometers for fluorescence and Raman microscopy: acousto-optic and liquid crystal tunable filters. Appl. Spectrosc., 48: 857-866.

NASA. 1973. Symposium on the Earth Resources Technology Satellite-1 (ERTS-1). Goddard Space Flight Center, Maryland, USA.

Schröck, E., du Manoir, S., Veldman, T., Schoell, B., Wienberg, J., Ferguson-Smith, M.A., Ning, Y., Ledbetter, D.H., Bar-Am, I., Soenksen, D., Garini, Y., and Ried, T. 1996. Multicolor spectral karyotyping of human chromosomes. Science, 273: 494-497.

Tsurui, H., Nishimura, H., Hattori, S., Hirose, S., Okumura, K., and Shirai, T. 2000. Seven-color fluorescence imaging of tissue samples based on Fourier spectroscopy and singular value decomposition. J. Histochem. Cytochem., 48: 653-662.

Wachman, E.S., Niu, W., and Farkas, D.L. 1997. AOTF microscope for imaging with increased speed and spectral versatility. Biophys. J., 73: $1215-1222$.

(Received for publication, November 1, 2002

and accepted, November 5, 2002) 